\title{
Long non-coding RNA CCAT1 is overexpressed in oral squamous cell carcinomas and predicts poor prognosis
}

\author{
GANESAN ARUNKUMAR ${ }^{1}$, AVANIYAPURAM KANNAN MURUGAN ${ }^{2}$, \\ HARIKRISHNAN PRASANNA SRINIVASA RAO ${ }^{3}$, SHANMUGAM SUBBIAH ${ }^{3}$, \\ RAMAMURTHY RAJARAMAN $^{3}$ and ARASAMBATTU KANNAN MUNIRAJAN ${ }^{1}$
}

\author{
${ }^{1}$ Department of Genetics, Dr ALM PG Institute of Basic Medical Sciences, University of Madras, \\ Taramani Campus, Chennai 600 113, India; ${ }^{2}$ Department of Molecular Oncology, King Faisal Specialist \\ Hospital and Research Center, Riyadh 11211, Saudi Arabia; ${ }^{3}$ Center for Oncology, Royapettah \\ Government Hospital and Kilpauk Medical College, Royapettah, Chennai 600 014, India
}

Received December 29, 2016; Accepted February 22, 2017

DOI: $10.3892 /$ br.2017.876

\begin{abstract}
Oral squamous cell carcinoma (SCC) is the most common malignant tumor in India with 5-year survival rates totaling $<50 \%$. Recently, dysregulation of non-coding RNA was reported as a potential hallmark of carcinogenesis. Colon Cancer Associated Transcript 1 (CCAT1), an 1ncRNA located in chromosome $8 \mathrm{q} 24$, close to the c-Myc gene, has been reported to be overexpressed in many human cancers. In the present study, the authors analyzed the expression of CCAT1, c-Myc and the miRNAs miR155-5p, let7b-5p, miR490-3p and miR218-5p sponged by CCAT1 in 60 oral tumor and 8 normal tissue samples by reverse transcription-quantitative polymerase chain reaction. CCAT1 was significantly overexpressed in $27 \%(16 / 60)$ of oral tumors. Interestingly, a high level of c-Myc expression was observed in all CCAT1 overexpressing cases $(\mathrm{P}=0.0473)$. Furthermore, CCAT1 overexpression significantly downregulated miR155-5p $(\mathrm{P}=0.03)$ and let7b-5p $(\mathrm{P}<0.0001)$. Oral cancer cases expressing high level of CCAT1 $(\mathrm{P}=0.01)$ presented poor therapeutic outcome. To the best of the authors' knowledge, this is the first study to report the overexpression of the CCAT1 in oral SCCs, and the results suggested that CCAT1 overexpression may sponge miR155-5p and let7b-5p, and may account for poor treatment response.
\end{abstract}

Correspondence to: Professor Arasambattu Kannan Munirajan, Department of Genetics, Dr ALM PG Institute of Basic Medical Sciences, University of Madras, Taramani Campus, Taramani, Chennai 600 113, India

E-mail: akmunirajan@gmail.com

Key words: non-coding RNA, IncRNA, colon cancer associated transcript 1 , oral cancer, competing endogenous RNA, tobacco, prognosis

\section{Introduction}

Oral squamous cell carcinoma (SCC) is the most common malignant tumor in India, and each year affects $\sim 300,000$ people worldwide (1). Most of the oral cancer cases in India are diagnosed with advanced clinical stage with 5-year survival rate $>50 \%$ (2). Tobacco chewing/smoking, alcohol consumption and human papillomavirus (HPV) infections, especially HPV 16 and 18 are the major risk factor for oral cancer (3). Oral carcinogenesis is a multistep process that involves the acquisition of many genetic and epigenetic alterations and dynamic changes in the gene expression of coding and non-coding RNAs.

Long non-coding RNAs are $>200$ nucleotides in length with limited or no protein-coding capacity, and serve primarily as the regulatory components in the cell. LncRNAs are reported as implicated in a vast number of cellular processes, such as cell proliferation, cell cycle progression, apoptosis, cell survival and motility. Dysregulation of several lncRNAs is related to metastasis, recurrence and prognosis in various cancer types (4). Enhanced expression of the IncRNA UCA1 has been suggested to promote cancer metastasis in head and neck cancers (5). Colon Cancer Associated Transcript 1 (CCAT1), an lncRNA, located in chromosome 8q24, close to c-Myc, a well-known oncogenic transcription factor (6). Previous studies have indicated that CCAT1 is differentially expressed in several types of cancers, including colon cancer, gastric cancer, gall bladder cancer and hepatocellular carcinoma (7-9). The CCAT1 was identified to be significantly overexpressed and clinically correlated with colon cancer patients' disease stage, lymph node metastasis and survival after surgery (10). In addition, CCAT1 was demonstrated to correlate with overall survival and progression-free survival in breast cancer (11). Although the CCAT1 has been reported to be involved in many human cancers, to date, its role in oral cancer is completely unknown.

Recently, lncRNAs have been indicated to function as a competing endogenous RNAs (ceRNA) by competitively binding to microRNAs (miRNAs) through their miRNA response elements (MRE) (12). These ceRNAs usually share 
MRE with other coding transcripts targeted by miRNAs and thereby act as sponges for that group of miRNAs, preventing the targeted mRNA transcripts from degradation. The upregulation of CCAT1 has been reported to downregulate the miRNAs miR 155-5p, let7b-5p, miR490-3p through a sponging mechanism and miR218-5p through epigenetic silencing (9,13-15). In the current report, the authors studied the role of CCAT1 in oral SCC by analyzing their expression and correlating the expression level with clinical characteristics. The expression of miR155-5p, let7b-5p, miR490-3p and miR218-5p was further analyzed in order to understand sponging activity of overexpressed CCAT1 and their role in oral carcinogenesis.

\section{Materials and methods}

Tumor tissue samples. The present study was approved by the Institutional Ethics Committee (IEC), Madras Medical College (Chennai, India; no. 04092010) and was conducted within the ethical framework of the Dr ALM PG Institute of Basic Medical Sciences (Chennai, India). A total of 60 oral cancer and 8 normal tissue samples were collected from Royapettah Government Hospital (Chennai, India). Tissue samples were maintained in RNAlater ${ }^{\circledR}$ solution (Ambion; Thermo Fisher Scientific, Inc., Waltham, MA, USA) and transported to the laboratory on ice. Patient's contextual and clinicopathological characteristics were documented with standard questionnaire as per the IEC guidelines, and written informed consent was obtained from each patient.

Isolation of RNA from tumor samples. Tissues stored in RNAlater ${ }^{\circledR}$ solution were washed twice with freshly prepared ice-cold PBS buffer. Tissue samples were homogenized in MicroSmash MS-100 automated homogenizer (Tomy, Tokyo, Japan) with zirconium beads for $1 \mathrm{~min}$ and the RNAeasy ${ }^{\circledR}$ mini kit (Qiagen GmbH, Hilden, Germany) was used for RNA extraction following supplier instructions.

Expression profiling by reverse transcription-quantitative polymerase chain reaction $(R T-q P C R)$. Expression profiling was performed for CCAT1 by using a custom-designed universal probe assay (Applied Biosystems; Thermo Fisher Scientific, Inc.). c-Myc was amplified by TaqMan assay (Applied Biosystems; Thermo Fisher Scientific, Inc.) and microRNAs by a custom-designed stem loop real-time assay (Applied Biosystems; Thermo Fisher Scientific, Inc.) (16). Briefly, cDNA synthesis for CCAT1 and GAPDH was conducted using custom-designed universal oligo reverse primers (Table I) (17) and using random hexamer primer (Sigma-Aldrich; Merck $\mathrm{KGaA}$, Darmstadt, Germany) for $\mathrm{c}-\mathrm{Myc}$, and specific stem-loop primers for each miRNA (Table I). Total RNA samples were pre-incubated at $65^{\circ} \mathrm{C}$ for $20 \mathrm{~min}$ to denature the RNA secondary structures, and were then placed on ice. All cDNA conversion reactions were conducted using reagents from the Invitrogen Reverse Transcription kit (Invitrogen; Thermo Fisher Scientific, Inc. USA) following the manufacturer's protocol. Following reverse transcription, the cDNAs were diluted 25 times and RT-qPCR was carried out in 384-well optical plates (in triplicate) in $10 \mu \mathrm{l}$ reactions with TaqMan $^{\circledR}$ 2X Universal Master mix (No AmpErase Uracil
N-Glycosylase; Thermo Fisher Scientific, Inc.), a specific forward primer, universal reverse primer and universal FAM-labelled MGB probe (Applied Biosystems; Thermo Fisher Scientific, Inc.) for miRNA/lncRNA, and a custom designed TaqMan ${ }^{\circledR}$ assay was used for c-Myc (Table II). Forward primers specific to the gene/miRNAs and universal reverse primers used for RT-qPCR are provided in Table II. The experiments were carried out using a 7900HT Real-Time PCR system (Applied Biosystems; Thermo Fisher Scientific, Inc.). A negative control without cDNA was also included in parallel for all assays. GAPDH was used as an endogenous control for lncRNA, and c-Myc and RNU44 as an endogenous control for miRNAs. Each experiment was conducted in triplicate and relative quantification of the expression was carried out using $2^{-\Delta \Delta C t}$ calculation (18).

Clinical evaluation. All 60 oral SCC patients were treated with 50-60 Gy radiation with concurrent three courses of cisplatin (Naprod Life Sciences, Mumbai, India) and 5-fluorouracil (Celon Laboratories Ltd, Hyderabad, India)]. The tumor response to this chemo/radio-therapy regimen was evaluated following 4 weeks of treatment. Patients who were evaluated as a complete and partial response to treatment were categorized as treatment responders, and the remaining patients were designated as poor responders. The patients were followed post-treatment for 13 months to study the tumor recurrence and therapeutic outcome.

Statistical analysis. The correlation between IncRNA CCAT1 expression and clinicopathological features of the patients were calculated by the chi-squared test and the differences between means were analyzed by Student's t-test (Mann-Whitney) using GraphPad Prism software (version, 6.0; GraphPad Software, Inc., La Jolla, CA, USA). Numerical data are presented as mean and \pm standard error of the mean in graphs. $\mathrm{P}<0.05$ was considered to indicate a statistically significant difference.

\section{Results}

Clinical characteristics. A total of 60 oral squamous cell carcinoma tumors and 8 normal tissue samples were used to study the expression of CCAT1. Of 60 oral SCC cases, a $42 \%(n=25)$ of the patients were $>50$ years of age and males were more predominant [(71.67\%) $n=43]$ than females $[(28.33 \% ; n=17)]$. Patients with any one or all habits (tobacco chewing/smoking and alcohol) account for $81.6 \%(n=49)$ of the cohort. $80 \%$ of the patients $(n=48)$ presented tumors grades of $>T 2$ stage and $86.7 \%(n=52)$ were positive for lymph node invasion. In the histological grading, $71.67 \%(n=43)$ of the cases were undifferentiated (Table III).

LncRNACCAT1 is overexpressed in oral SSCs. Expression level of CCAT1 was analyzed in 60 oral tumors and 8 normal tissue samples by RT-qPCR. CCAT1 overexpression was observed in $27 \%(16 / 60)$ of oral cancer samples (Fig. 1) and the expression was identified to be exponentially increased across the tumor samples. Therefore, the CCAT1 overexpressed samples were characterized into three categories, based on the expression levels. In group 1, the mean CCAT1 expression level was equal to the normal samples $[73 \%(44 / 60)]$, in group 2 the expression 
Table I. List of reverse transcription primers used for cDNA synthesis.

\begin{tabular}{ll} 
Gene & \multicolumn{1}{c}{ Primer sequence $\left(5^{\prime}-3^{\prime}\right)$} \\
\hline Oligo primer for mRNA/lncRNA & CAGTGCAGGGTCCGAGGTACAGAGCCACCTGGGCAA \\
RNU44 & TTTTTTTTTVN \\
hsa-miR-let7b-5p & TTTGCACTGGATACGACAGTCAG \\
hsa-miR-218-5p & GTCGTATCCAGTGCGTCGAGTGACACGAGAGCCACCTGGGCAA \\
& TTTGCACTGGATACGACAACCAC \\
hsa-miR-490-3p & GTCGTATCCAGTGCGTCGAGTGACACGAGAGCCACCTGGGCAA \\
& TTTGCACTGGATACGACACATGG \\
hsa-miR-155-5p & GTCGTATCCAGTGCGTCGAGTGACACGAGAGCCACCTGGGCAA \\
& TTTGCACTGGATACGACCAGCAT \\
& GTCGTATCCAGTGCGTCGAGTGACACGAGAGCCACCTGGGCAA \\
& TTTGCACTGGATACGACACCCCT
\end{tabular}

$\mathrm{V},(\mathrm{A}, \mathrm{C}$ or $\mathrm{G}) ; \mathrm{N},(\mathrm{A}, \mathrm{C}, \mathrm{G}$ or T); miR, microRNA.

Table II. List of gene specific primers and sequences of universal primer and probe used in RT-qPCR.

\begin{tabular}{ll}
\hline Gene & \\
\hline CCAT1 & Forward primer $\left(5^{\prime}-3^{\prime}\right)$ \\
GAPDH & GTGTATCTTAGTTCAACCAAATTGTAATCATCTG \\
RNU44 & GAAGAGGGGAGGGGCCTAGG \\
let7b-5p & GCAAATGCTGACTGAACATGA \\
miR-218-5p & GTGAGGTAGTAGGTTGTGTG \\
miR-490-3p & CAGTTGTGCTTGATCTAACCA \\
miR-155-5p & CAACCTGGAGGACTCCAT \\
Universal reverse primer used for lncRNA/mRNA & CGCAGTTAATGCTAATCGTGATAG \\
Universal reverse primer used for miRNA/snoRNA & CAGTGCAGGGTCCGAGGT \\
c-Myc & TCGTATCCAGTGCGTCGAGT \\
Universal probe for RT-qPCR assay & Forward: ATTCTCTGCTCTCCTCGACG \\
\end{tabular}

RT-qPCR, reverse transcription-quantitative polymerase chain reaction; miR, microRNA; CCAT1,CCAT1,Colon CancerAssociated Transcript 1.

was $>15$ fold higher than normal tissues $[13.5 \%(8 / 60)]$ and in group 3 the expression was $>30$-fold higher than normal tissues [13.5\% (8/60)]. Group 2 and group 3 tumors presented statistically significant overexpression of CCAT1 $(\mathrm{P}=0.0002$ and $\mathrm{P}=0.001$, respectively), when compared to control tissue samples.

Association of CCAT1 with clinicopathological characteristics. In order to understand the significance of lncRNA CCAT1 overexpression in oral cancer, CCAT1 overexpression was correlated with clinicopathological features of the tumors. In univariate analysis, except for age $(\mathrm{P}=0.0072)$, none of the clinicopathological features tested were statistically significant (Table IV), and this may be due to the wide variation in the expression level of CCAT1 in tumor samples of the clinical sub-groups. Patients with higher tumor grade and undifferentiated pathology presented high level expression of CCAT1. The majority of the CCAT1 overexpressing samples $(62.5 \%)$ were tumors from buccal mucosa and the tumor samples with tobacco smoking and practising both the habit (tobacco chewing and smoking) expressed a high level of CCAT1. The authors then analyzed the head and neck cancer datasets from TCGA database using the TANRIC interactive platform (16) and a similar CCAT1 overexpression was observed in cases with the history of tobacco abuse $(\mathrm{P}<0.0001)$, suggesting the role of tobacco smoking/chewing in oral tumorigenesis.

CCAT1 overexpression is associated with treatment response. The CCAT1 overexpression has been indicated to be associated with the clinical features and therapeutic response in various human cancers $(10,11)$. Therefore, the authors correlated the 
Table III. Relationship between CCAT1 expression and clinicopathological characteristics in oral cancer patients.

\begin{tabular}{|c|c|c|c|c|}
\hline \multirow[b]{2}{*}{ Clinical characters } & \multirow[b]{2}{*}{ No. } & \multicolumn{2}{|c|}{ CCAT1 expression level } & \multirow[b]{2}{*}{ P-value ${ }^{a}$} \\
\hline & & Low $(n=44)$ & $\operatorname{High}(\mathrm{n}=16)$ & \\
\hline Age & & & & 0.5558 \\
\hline$<50$ & 25 & 17 & 8 & \\
\hline$\geq 51$ & 35 & 27 & 8 & \\
\hline Gender & & & & 0.1199 \\
\hline Male & 43 & 29 & 14 & \\
\hline Female & 17 & 15 & 2 & \\
\hline Risk habits & & & & 1.0000 \\
\hline Positive & 49 & 36 & 13 & \\
\hline Negative & 11 & 8 & 3 & \\
\hline Tumor stage & & & & 1.0000 \\
\hline$\leq \mathrm{T} 2$ & 12 & 9 & 3 & \\
\hline$>\mathrm{T} 2$ & 48 & 35 & 13 & \\
\hline Nodal invasion & & & & 0.4293 \\
\hline Positive & 52 & 39 & 13 & \\
\hline Negative & 8 & 5 & 3 & \\
\hline Differentiation & & & & 0.5181 \\
\hline Differentiated & 17 & 14 & 3 & \\
\hline Undifferentiated & 43 & 30 & 13 & \\
\hline
\end{tabular}

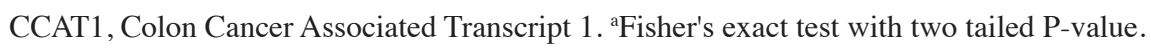

CCAT1 expression level with the patient's treatment responses. Most of the patients participated in the presented study are from rural areas and are uneducated, and 50 of 60 patients failed to appear for the monthly check up. Only 10 patients were followed-up post-treatment for 13 months to analyze the treatment response. As illustrated in Fig. 2A, patients with significant overexpression of CCAT1 presented a poor therapeutic response $(\mathrm{P}=0.01)$. Furthermore, when overall survival of the high/low level of CCAT1 expressing group of head and neck cases from the TCGA database was analyzed, a high level expression of CCAT1 presented strong association with poor survival $(\mathrm{P}=0.008$; data not shown).

The c-Myc oncogene is overexpressed in oral squamous cell carcinomas. To understand the functional significance of c-Myc, an oncogenic transcription factor reported to be associated with various cancers including oral cancer and located in the same chromosome loci with CCAT1 $(7,19,20)$, c-Myc expression was analyzed in all samples. Interestingly, c-Myc was demonstrated as overexpressed in the tumor samples that expressed a high level of CCAT1 ( $\mathrm{P}=0.0473$; Fig. 2B).

Expression of miR155-5p, let $7 b-5 p$, miR218-5p and miR490-3p in oral SSCs. CCAT1 was reported to function as ceRNA in various cancers (13-15). Therefore, to understand whether a CCAT1-mediated ceRNA network is functioning in oral SCCs, the expression of miR155-5p, let7b-5p, miR218-5p and miR490-3p was profiled and a significant downregulation of miR155-5p $(\mathrm{P}=0.037)$ and let7b-5p $(\mathrm{P}<0.0001)$ was observed in oral SCCs compared to normal tissues (Fig. 3A). The miRNAs, miR218-5p and miR490-3p were upregulated in oral SCC tumors compared with normal samples but it was identified as being downregulated significantly in CCAT1 overexpressing tumor group 2 and 3 (Fig. 3B). let7b-5p was significantly downregulated across all the tumor groups (group $1, \mathrm{P}=0.0001$; group 2, $\mathrm{P}=0.004$; and group $3, \mathrm{P}=0.0002$ ) compared with the normal tissues (Fig. 3C). Consistent with the previous reports, a downregulation of let7b-5p, and miR155-5p was observed in tumor samples that overexpressed CCAT1, compared with normal tissues. These results suggested that CCAT1 acts as sponge for miR155-5p, let7b-5p, miR218-5p and miR490-3p, resulting in the degradation of these microRNAs.

\section{Discussion}

lncRNAs are $>200 \mathrm{nt}$ in length and serves critical regulatory roles in diverse cellular processes. Differential expression of lncRNAs is increasingly documented as a hallmark feature in cancer (7-9). In the present study, the authors analyzed the expression level of 1ncRNA CCAT1 and explored its clinical significance in oral cancer. It was identified that CCAT1 expression was several folds higher in oral cancer samples, when compared with the normal tissue samples. Interestingly, as the expression of CCAT1 in tumor samples is demonstrated to be exponentially increased, the tumors were characterized into three groups in the increasing order of expression and correlated the CCAT1 expression levels with patients' 
A

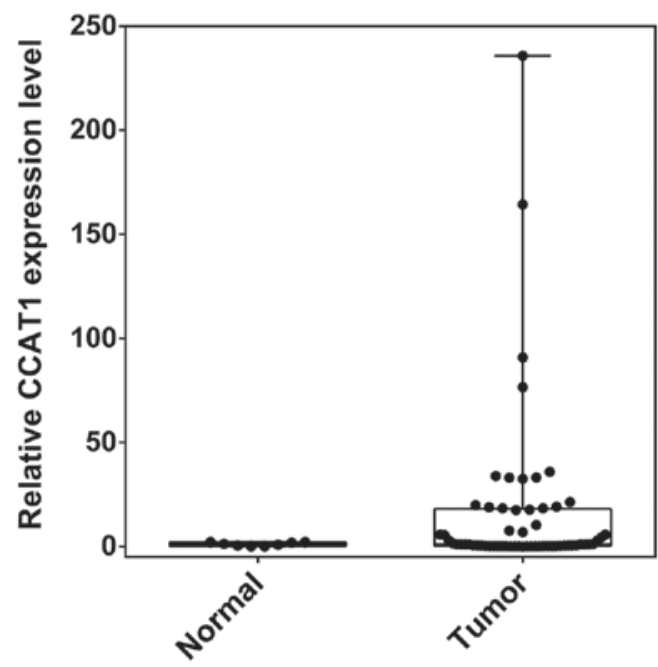

B

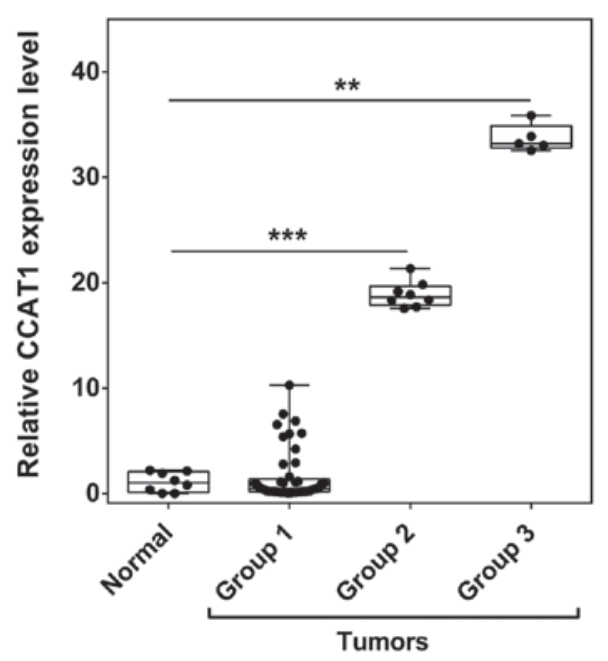

E

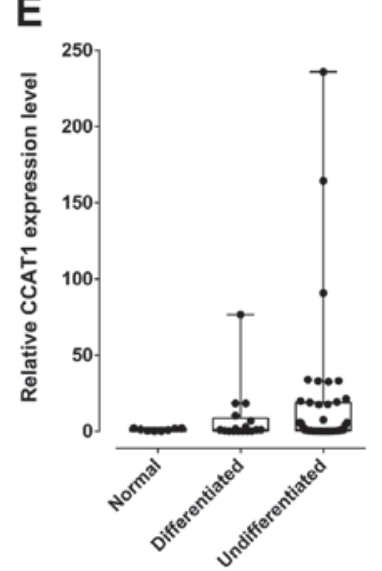

Figure 1. Expression of CCAT1 in oral squamous cell carcinomas. (A) Expression of CCAT1 in oral tumors compared with normal tissues. (B) Expression of CCAT1 was identified to be exponentially higher in oral tumors. The samples are categorized into groups depending on the expression clusters. (C) Oral cancer patients with a smoking habit presented a higher, but not significant, expression of CCAT1 compared to those who chew tobacco. (D) CCAT1 was overexpressed in tumors with tumor grade $>\mathrm{T} 2$, compared with normal tissues. (E) Undifferentiated oral tumors expressed higher levels of CCAT1, when compared with tumors of differentiated cellular pathology. The groups used in the study are as follows: Normal, unaffected tissue samples (n=8); group 1 , mean CCAT1 expression level=mean CCAT1 expression level in the normal samples $(n=44)$; group 2, the mean CCAT1 expression was $>15$-fold higher than normal tissues $(n=8)$; group 3, mean CCAT1 expression was $>30$-fold higher than normal tissues $(n=8)$. Data are presented as the mean \pm standard error of the mean. ${ }^{* *} \mathrm{P}<0.01,{ }^{* * *} \mathrm{P}<0.001$ vs. normal tissues. CCAT1, Colon Cancer Associated Transcript 1.

clinicopathological features. The CCAT1 amplified samples congregated into the tumors with aggressive phenotypes and the CCAT1 overexpression was observed in the patients with cigarette smoking $(\mathrm{P}=0.68)$. Consistent with these results, head and neck cancer datasets from TCGA database also presented overexpression of CCAT1 in tumors with tobacco smoking history. Furthermore, patients with overexpression of CCAT1 demonstrated a poor response to treatment $(\mathrm{P}=0.01$; Figs. 1 and 2A) and similar results were also presented in the head and neck cancer TCGA datasets. Copy number alterations of CCAT1 have been reported to be frequent in breast cancer (21) and, in ER ${ }^{+} / \mathrm{HER} 2-$ cases, CCAT1 amplification was reported to be strongly associated with early relapse (22).

Myc has been demonstrated to serve a key role in the progression of cell proliferation and tumorigenesis in oral cancer (23). As the c-Myc gene is located $515 \mathrm{~kb}$ upstream of CCAT1 in the same chromosome loci, the authors tested the c-Myc expression and observed significant high level of
c-Myc in CCAT1 overexpressing tumors. To the best of the authors' knowledge, the present study was the first to observe the concomitant overexpression of both c-Myc and CCAT1 in oral cancer, although the c-Myc protein has already been reported as overexpressed in $80 \%$ of the oral SCCs and correlated with poor prognosis (24). Furthermore, c-Myc was reported to contribute microenvironment-mediated drug resistance in AML and oral cell lines $(25,26)$. The CCAT1 locus has been presented to harbor a super-enhancer that could control the c-Myc expression. The CCAT1 was demonstrated to interact with a transcriptional enhancer MYC-335 via chromatin looping and consequently, could interact with the MYC-promoter resulting regulation of c-Myc expression in cis $(10,27)$. In addition, knockdown of CCAT1 led to a decrease in c-Myc levels in colon cancer-derived cell lines (28). In addition, aberrant expression of CCAT1 controlled by c-Myc predicted the poor prognosis in hepatocellular carcinoma and gastric cancer $(29,30)$. 
Table IV. Relationship between CCAT1 expression categories in groups and their clinicopathological characteristics in oral cancer patients.

\begin{tabular}{|c|c|c|c|c|}
\hline \multirow[b]{2}{*}{ Clinical characteristics } & \multicolumn{3}{|c|}{ CCAT1 expression categories } & \multirow[b]{2}{*}{ P-value } \\
\hline & $\begin{array}{c}\text { Group 1 } \\
(\mathrm{n}=44)(\%)\end{array}$ & $\begin{array}{c}\text { Group } 2 \\
(\mathrm{n}=8)(\%)\end{array}$ & $\begin{array}{c}\text { Group } 3 \\
(\mathrm{n}=8)(\%)\end{array}$ & \\
\hline Age & & & & $0.0072^{\mathrm{b}}$ \\
\hline$<50$ & $17(38.6)$ & $7(87.5)$ & $1(12.5)$ & \\
\hline$\geq 51$ & $27(61.4)$ & $1(12.5)$ & $7(87.5)$ & \\
\hline Gender & & & & 0.2601 \\
\hline Male & $29(66)$ & $7(87.5)$ & $7(87.5)$ & \\
\hline Female & $15(44)$ & $1(12.5)$ & $1(12.5)$ & \\
\hline Risk habits & & & & 0.1526 \\
\hline Positive & $36(81.9)$ & $5(62.5)$ & $8(100)$ & \\
\hline Negative & $8(18.1)$ & $3(37.5)$ & $0(-)$ & \\
\hline Tumor stage & & & & 0.7804 \\
\hline$\leq \mathrm{T} 2$ & $9(20.5)$ & $1(12.5)$ & $1(12.5)$ & \\
\hline$>\mathrm{T} 2$ & $35(79.5)$ & $7(87.5)$ & $7(87.5)$ & \\
\hline Nodal invasion & & & & 0.5784 \\
\hline Positive & $39(88.6)$ & $7(87.5)$ & $6(75)$ & \\
\hline Negative & $5(11.4)$ & $1(12.5)$ & $2(25)$ & \\
\hline Differentiation & & & & 0.1723 \\
\hline Differentiated & $14(31.8)$ & $2(25)$ & $0(-)$ & \\
\hline Undifferentiated & $30(68.2)$ & $6(75)$ & $8(100)$ & \\
\hline
\end{tabular}

Normal, unaffected tissue samples ( $\mathrm{n}=8$ ); group 1, mean CCAT1 expression level=mean CCAT1 expression level in the normal samples $(\mathrm{n}=44)$; group 2, the mean CCAT1 expression was $>15$-fold higher than normal tissues $(n=8)$; group 3, mean CCAT1 expression was $>30$-fold higher than normal tissues $(\mathrm{n}=8)$. ${ }^{\mathrm{a}} \mathrm{Chi}$-squared test with two tailed P-values. ${ }^{\mathrm{b}}$ Statistically significant. CCAT1, Colon Cancer Associated Transcript 1.

A

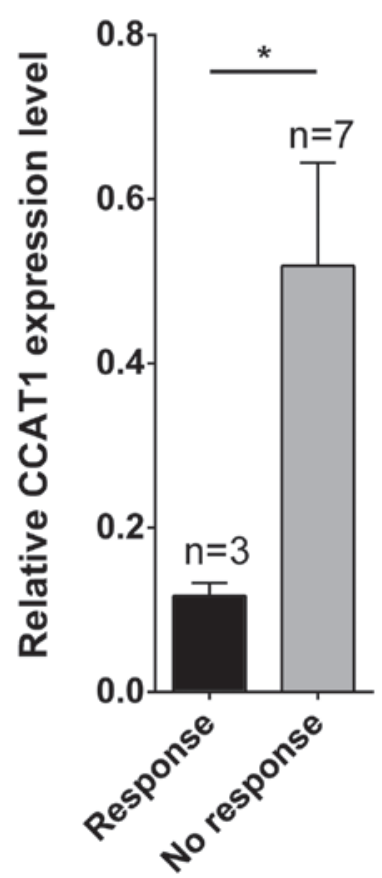

B

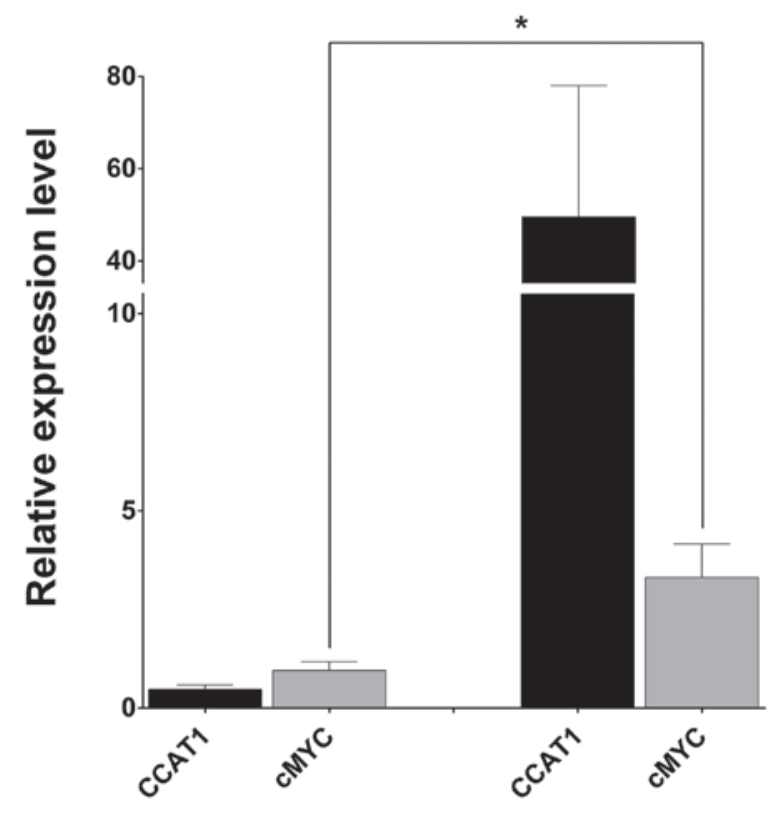

Figure 2. c-Myc and CCAT1 are concomitantly overexpressed in oral squamous cell carcinomas and are associated with clinical outcome. (A) Oral cancer patients overexpressed CCAT1 presented poor treatment outcome. (B) Oral tumors overexpressed CCAT1 also significantly expressed c-Myc at higher levels. Data are presented as the mean \pm standard error of the mean. ${ }^{*} \mathrm{P}<0.05$. CCAT1, Colon Cancer Associated Transcript 1. 
A

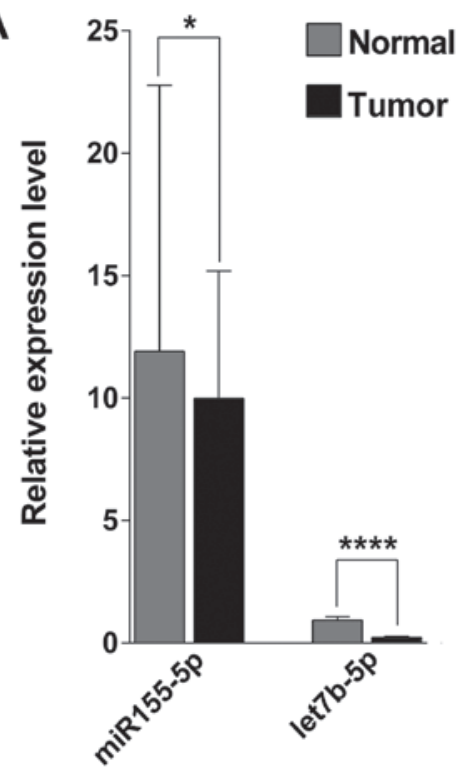

C

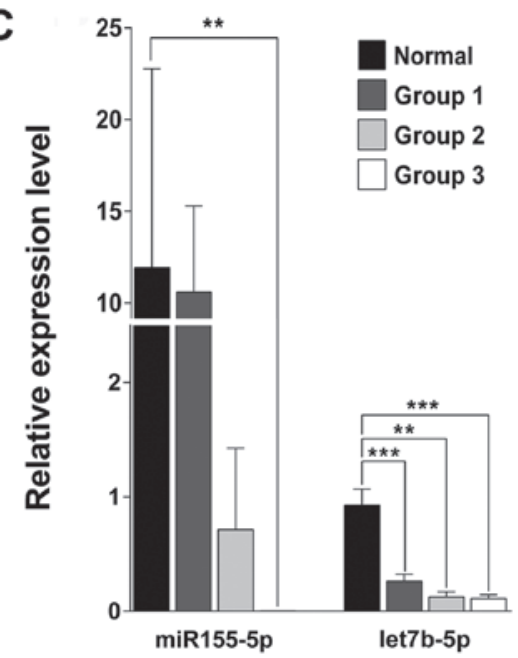

B

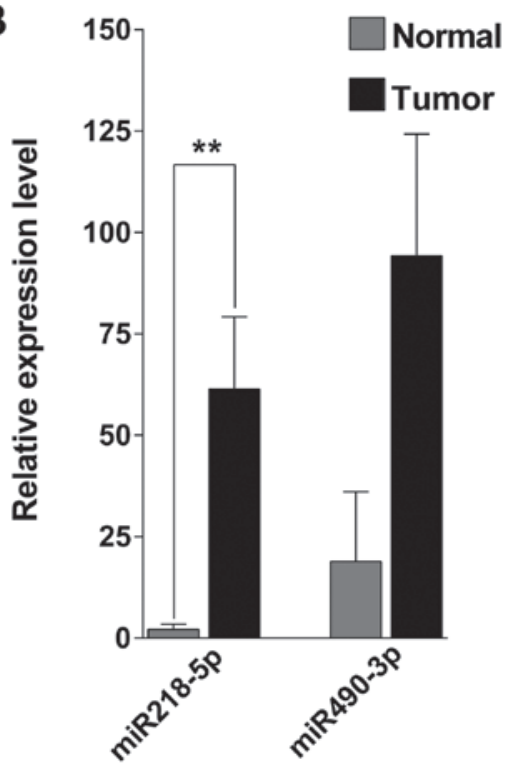

D

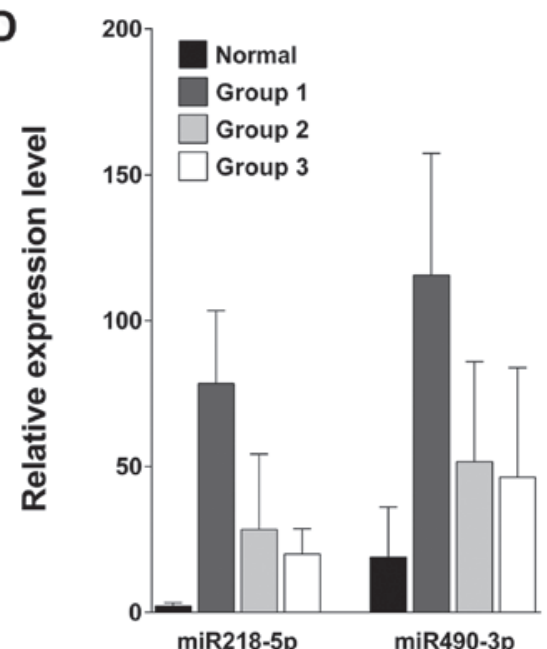

Figure 3. Expression of miR155-5p, let7b-5p, miR218-5p and miR490-3p. (A) miR155-5p and let7b-5p were significantly downregulated in oral tumors, when compared with normal tissues. (B) miR218-5p and miR490-3p were upregulated in oral tumors, when compared with normal tissues. (C) miR155 and let7b were downregulated in oral tumor groups and miR-let7b was significantly downregulated in all three groups of the CCAT1 overexpressed samples, when compared with the normal tissues. (D) miR218 and miR490 were downregulated in tumor groups overexpressing CCAT1, but were overexpressed when compared with normal tissue levels. Notably, group 1 samples expressed CCAT1 at levels equal to normal, group 2 samples expressed CCAT1 at 15 -fold higher and group 3 samples expressed CCAT1 at $>30$-fold. The groups used in the study are as follows: Normal, unaffected tissue samples (n=8); group 1, mean CCAT1 expression level=mean CCAT1 expression level in the normal samples ( $\mathrm{n}=44)$; group 2, the mean CCAT1 expression was $>15$-fold higher than normal tissues $(\mathrm{n}=8)$; group 3, mean CCAT1 expression was $>30$-fold higher than normal tissues $(\mathrm{n}=8)$. Data are presented as the mean \pm standard error of the mean. ${ }^{*} \mathrm{P}<0.05,{ }^{* * *} \mathrm{P}<0.01,{ }^{* * *} \mathrm{P}<0.001,{ }^{* * * *} \mathrm{P}<0.0001$ vs. normal tissues. CCAT1, Colon Cancer Associated Transcript 1; miR, microRNA.

Functioning as ceRNA, CCAT1 has been reported to downregulate miR $155-5 p$, let7b-5p and miR490-3p through sequestration and miR $218-5 \mathrm{p}$ by epigenetic regulation $(9,13-15)$. Therefore, the expression of miR155-5p, let7b-5p, miR218-5p and miR490-3p in oral tumors and normal tissues was analyzed. Interestingly, miR155-5p and let7b-5p were significantly downregulated in all four tumor groups compared with normal tissues, but miR218-5p and miR490-3p only in tumor groups 2 and 3 (Fig. 3). In lung cancer, CCAT1 was reportedly overexpressed in cigarette smokers, and indicated to alter the cell cycle transition through epigenetic silencing of miR281 and activation of EMT-associated gene BMI1 (13). CCAT1 was reported to promote hepatocellular carcinoma progression by sponging the microRNA let7 family, tumor suppressor
miRNAs well associated with oral cancer (9). The CCAT1 has been reported to regulate miR490 in gastric cancer by similar mechanism mentioned above and favors tumor cell migration and accounts for poor therapeutic outcome (14). The presented results suggested that CCAT1 overexpression was likely to affect the downstream target miRNA level in oral tumors via sponging mechanism as shown in other cancers $(9,14)$. In addition, CCAT1 overexpressed samples expressed a low level of miR 155 and let $7 \mathrm{~b}$ and the same has been frequently reported to be deregulated in several cancer cell lines and cancers (31). All these observations suggest that CCAT1 overexpression may serve an important role in oral carcinogenesis and treatment response. Further research is warranted to confirm CCAT1 as a potential target for oral cancer diagnosis and treatment. 


\section{Acknowledgements}

The authors would like to thank the patients for their consent to provide clinical samples. G.A. gratefully acknowledges the University Grant Commission (UGC), Government of India for providing research fellowship. The authors also gratefully acknowledge the Department of Science and Technology Fund for Improvement of S\&T Infrastructure, UGC-Special Assistance Programme and the Department of Health Multidisciplinary Research Unit infrastructure facility. The study was supported by grants received by the corresponding authorfrom theDepartment of AtomicEnergy,Board of Research in Nuclear Sciences (grant no. 35/14/10/2014-BRNS/0210), and the Department of Biotechnology, Government of India (grant no. BT/PR4820/MED/12/622/2013).

\section{References}

1. Torre LA, Bray F, Siegel RL, Ferlay J, Lortet-Tieulent J and Jemal A: Global cancer statistics, 2012. CA Cancer J Clin 65: 87-108, 2015.

2. Bourhis J, Overgaard J, Audry H, Ang KK, Saunders M, Bernier J, Horiot JC, Le Maître A, Pajak TF, Poulsen MG, et al: Hyperfractionated or accelerated radiotherapy in head and neck cancer: A meta-analysis. Lancet 368: 843-854, 2006.

3. Manikandan M, Deva Magendhra Rao AK, Arunkumar G, Manickavasagam M, Rajkumar KS, Rajaraman R and Munirajan AK: Oral squamous cell carcinoma: microRNA expression profiling and integrative analyses for elucidation of tumourigenesis mechanism. Mol Cancer 15: 28, 2016.

4. Mercer TR, Dinger ME and Mattick JS: Long non-coding RNAs: Insights into functions. Nat Rev Genet 10: 155-159, 2009.

5. Fang Z, Wu L, Wang L, Yang Y, Meng Y and Yang H: Increased expression of the long non-coding RNA UCA1 in tongue squamous cell carcinomas: A possible correlation with cancer metastasis. Oral Surg Oral Med Oral Pathol Oral Radiol 117: 89-95, 2014.

6. Alaiyan B, Ilyayev N, Stojadinovic A, Izadjoo M, Roistacher M, Pavlov V, Tzivin V, Halle D, Pan H, Trink B, et al: Differential expression of colon cancer associated transcript1 (CCAT1) along the colonic adenoma-carcinoma sequence. BMC Cancer 13: 196 2013.

7. Yang F, Xue X, Bi J, Zheng L, Zhi K, Gu Y and Fang G: Long noncoding RNA CCAT1, which could be activated by c-Myc, promotes the progression of gastric carcinoma. J Cancer Res Clin Oncol 139: 437-445, 2013.

8. Ma MZ, Chu BF, Zhang Y, Weng MZ, Qin YY, Gong W and Quan ZW: Long non-coding RNA CCAT1 promotes gallbladder cancer development via negative modulation of miRNA-218-5p. Cell Death Dis 6: e1583, 2015.

9. Deng L, Yang SB, Xu FF and Zhang JH: Long noncoding RNA CCAT1 promotes hepatocellular carcinoma progression by functioning as let-7 sponge. J Exp Clin Cancer Res 34: 18, 2015.

10. He X, Tan X, Wang X, Jin H, Liu L, Ma L, Yu H and Fan Z: C-Myc-activated long noncoding RNA CCAT1 promotes colon cancer cell proliferation and invasion. Tumour Boil 35: 12181-12188, 2014

11. Zhang XF, Liu T, Li Y and Li S: Overexpression of long non-coding RNA CCAT1 is a novel biomarker of poor prognosis in patients with breast cancer. Int J Clin Exp Pathol 8: 9440-9445, 2015.

12. Salmena L, Poliseno L, Tay Y, Kats L and Pandolfi PP: A ceRNA hypothesis: The Rosetta stone of a hidden RNA language? Cell 146: 353-358, 2011

13. Lu L, Xu H, Luo F, Liu X, Lu X, Yang Q, Xue J, Chen C, Shi L and Liu Q: Epigenetic silencing of miR-218 by the lncRNA CCAT1, acting via BMI1, promotes an altered cell cycle transition in the malignant transformation of HBE cells induced by cigarette smoke extract. Toxicol Appl Pharmacol 304: 30-41, 2016.
14. Zhou B, Wang Y, Jiang J, Jiang H, Song J, Han T, Shi J and Qiao H: The long noncoding RNA colon cancer-associated transcript-1/miR-490 axis regulates gastric cancer cell migration by targeting hnRNPA1. IUBMB Life 68: 201-210, 2016.

15. Chen L, Wang W, Cao L, Li Z and Wang X: Long Non-Coding RNA CCAT1 acts as a competing endogenous RNA to regulate cell growth and differentiation in acute myeloid leukemia. Mol Cells 39: 330-336, 2016

16. Chen C, Ridzon DA, Broomer AJ, Zhou Z, Lee DH, Nguyen JT, Barbisin M, Xu NL, Mahuvakar VR, Andersen MR, et al: Real-time quantification of microRNAs by stem-loop RT-PCR. Nucleic Acids Res 33: e179, 2005.

17. Kang K, Zhang X, Liu H, Wang Z, Zhong J, Huang Z, Peng X, Zeng Y, Wang Y, Yang Y, et al: A novel real-time PCR assay of microRNAs using S-Poly(T), a specific oligo(dT) reverse transcription primer with excellent sensitivity and specificity. PLoS One 7: e48536, 2012.

18. Schmittgen TD and Livak KJ: Analyzing real-time PCR data by the comparative C(T) method. Nat Protoc 3: 1101-1108, 2008.

19. Li J, Han L, Roebuck P, Diao L, Liu L, Yuan Y, Weinstein JN and Liang H: TANRIC: An interactive open platform to explore the function of lncRNAs in cancer. Cancer Res 75: 3728-3737, 2015.

20. Grisanzio C and Freedman ML: Chromosome 8q24-Associated cancers and MYC. Genes Cancer 1: 555-559, 2010.

21. Naylor TL, Greshock J, Wang Y, Colligon T, Yu QC, Clemmer V, Zaks TZ and Weber BL: High resolution genomic analysis of sporadic breast cancer using array-based comparative genomic hybridization. Breast Cancer Res 7: R1186-R1198, 2005.

22. Bilal E, Vassallo K, Toppmeyer D, Barnard N, Rye IH, Almendro V, Russnes H, Børresen-Dale AL, Levine AJ, Bhanot $\mathrm{G}$ and Ganesan S: Amplified loci on chromosomes 8 and 17 predict early relapse in ER-positive breast cancers. PLoS One 7: e38575, 2012.

23. Mishra R and Das BR: Early overexpression of Cdk4 and possible role of KRF and c-myc in chewing tobacco mediated oral cancer development. Mol Biol Rep 30: 207-213, 2003.

24. Pai R, Pai S, Lalitha R, Kumaraswamy S, Lalitha N, Johnston R and Bhargava M: Over-expression of c-Myc oncoprotein in oral squamous cell carcinoma in the South Indian population. Ecancermedicalscience 3: 128, 2009.

25. Xia B, Tian C, Guo S, Zhang L, Zhao D, Qu F, Zhao W, Wang Y, Wu X, Da W, et al: c-Myc plays part in drug resistance mediated by bone marrow stromal cells in acute myeloid leukemia. Leuk Res 39: 92-99, 2015.

26. Ghosh RD, Ghuwalewala S, Das P, Mandloi S, Alam SK, Chakraborty J, Sarkar S, Chakrabarti S, Panda CK and Roychoudhury S: MicroRNA profiling of cisplatin-resistant oral squamous cell carcinoma cell lines enriched with cancer-stem-cell-like and epithelial-mesenchymal transition-type features. Sci Rep 6: 23932, 2016.

27. Pomerantz MM, Ahmadiyeh N, Jia L, Herman P, Verzi MP, Doddapaneni H, Beckwith CA, Chan JA, Hills A, Davis M, et al: The 8q24 cancer risk variant rs6983267 shows long-range interaction with MYC in colorectal cancer. Nat Genet 41: 882-884, 2009.

28. Xiang JF, Yin QF, Chen T, Zhang Y, Zhang XO, Wu Z, Zhang S, Wang HB, Ge J, Lu X, et al: Human colorectal cancer-specific CCAT1-L lncRNA regulates long-range chromatin interactions at the MYC locus. Cell Res 24: 513-531, 2014.

29. Zhang Y, Ma M, Liu W, Ding W and Yu H: Enhanced expression of long noncoding RNA CARLo-5 is associated with the development of gastric cancer. Int J Clin Exp Pathol 7: 8471-8479, 2014.

30. Zhu HQ, Zhou X, Chang H, Li HG, Liu FF, Ma CQ and Lu J: Aberrant expression of CCAT1 regulated by c-Myc predicts the prognosis of hepatocellular carcinoma. Asian Pac J Cancer Prev 16: 5181-5185, 2015.

31. Li CL, Nie H, Wang M, Su LP, Li JF, Yu YY, Yan M, Qu QL, Zhu ZG and Liu BY: MicroRNA-155 is downregulated in gastric cancer cells and involved in cell metastasis. Oncol Rep 27: 1960-1966, 2012. 